\title{
Evaluación de la madurez neuropsicológica infantil en preescolares
}

\author{
Children neuropsychological assessment in preschoolers
}

Javier Humberto Parra-Pulido', Lucía Carlota Rodríguez-Barreto², Julián David Chinome-Torres³

1 Psicólogo. Joven Investigador UPTC Grupo de Investigación GIPCS. Tunja, Colombia. e-mail: javierhumberto.parra@uptc.edu.co

2 Psicóloga. Doctora en Neurociencias. Docente Titular Escuela de Psicología, Grupo de Investigación GIPCS. Universidad Pedagógica y Tecnológica de Colombia. Tunja, Colombia. e-mail: lucia.rodriguezb@uptc.edu.co

3 Grupo de Investigación GIPCS. Tunja, Colombia. e-mail: juliandavid.chinome@uptc.edu.co

\begin{abstract}
Resumen
Introducción: La carencia de programas de intervención neuropsicológica en población preescolar es una tarea pendiente en Colombia, siendo la etapa de los tres a los seis años, un periodo crítico en el desarrollo y adquisición de habilidades cognitivas y sociales a lo largo del ciclo vital. Objetivo: Se buscó evaluar la madurez neuropsicológica en niños (as) de 3 a 6 años de un colegio privado de la ciudad de Tunja, con el fin de diseñar un programa de corrección neuropsicológica. Materiales y métodos: Se realizó un estudio observacional- descriptivo y comparativo en 46 niños (as) con edad promedio de 57 meses, teniendo como base las funciones mentales del neurodesarrollo propuestas por Portellano, Mateos y Martínez y evaluadas con el CUMANIN y una historia clínica. Los datos recibieron un tratamiento estadístico de cálculo de medias y desviaciones a partir del valor promedio de la prueba adaptada al contexto colombiano, un análisis a través del estadístico T de Student con las puntuaciones obtenidas en la aplicación y una comparación entre resultados por grupo de edad. Resultados: Los resultados son inferiores en el grupo de edad de 55- 78 meses respecto al grupo de edad de 36 a 42 meses. No se obtuvieron diferencias significativas por sexo. El 63\% de los participantes presentó puntuaciones por debajo de las esperadas en por lo menos dos de las áreas evaluadas. Conclusión: A partir de los datos obtenidos se justifica el diseño de un programa de corrección neuropsicológica.
\end{abstract}

Palabras clave: Neuropsicología; desarrollo de programa; desarrollo infantil. (Fuente: DeCS, Bireme).

\begin{abstract}
Introduction: The lack of neuropsychological intervention programs in preschool population is a pending task in Colombia, where the stage from 3 to 6 years old is a critical period in the development and acquisition of cognitive and social skills throughout the life cycle. Objective: To evaluate the neuropsychological maturity in children from 3 to 6 years old in a private school in the city of Tunja in order to design a program of neuropsychological correction. Materials and methods: An observational, descriptive and comparative study was conducted in 46 children with an average age of 57 months on the basis of the mental functions of the neurodevelopment proposed by Portellano, Mateos and Martínez and was evaluated using CUMANIN and a medical history. The data received a statistical treatment of calculating the averages and deviations from the average value of the test adapted to the Colombian context, an analysis using the Statistical T of Student with the results obtained in the application and a comparison of results by age group. Results: The results indicate lower scores in the age group of 55-78 months compared to the age group of 36 to 42 months. No significant differences by gender were found. $63 \%$ of the participants obtained scores
\end{abstract}


below the expected in at least two of the areas evaluated. Conclusion: The data obtained justify the design of a neuropsychological correction program.

Keywords: Neuropsychology; program development; child development. (Source: DeCS, Bireme).

\section{Introducción}

El estudio de la neuropsicología infantil, enmarca la temporalidad como factor inherente vinculado al desarrollo y la adquisición de habilidades cognoscitivas, dando lugar a la Madurez Neuropsicológica (MN), considerada como la respuesta a patrones evolutivos propios del desarrollo normal del individuo, vinculado a la edad cronológica en relación con las funciones cognoscitivas específicas.1-3 Además de ser biológico, los factores culturales y sociales propician efectos potencializadores sobre el desarrollo neuropsicológico en cuanto sea un espacio facilitador y estimulador para generar las condiciones cognitivas, adaptativas y comportamentales propicias para su evolución, siendo la edad entre tres y seis años según Portellano, et al.,4 el periodo fundamental para que se dé un desarrollo óptimo en la adquisición de funciones mentales superiores.

La importancia de estudiar el neurodesarrollo infantil, está enmarcado en la necesidad de brindar una buena calidad de vida al individuo, el cual se podrá desenvolver en las diferentes áreas de desarrollo a lo largo de la vida. Sin embargo, se estima que entre el 10 y el $15 \%$ de la población infantil en países subdesarrollados padece de algún tipo de alteración en el neurodesarrollo en diferentes grados, incluyendo desde problemas en el aprendizaje hasta parálisis cerebral.5

Estas alteraciones afectarán a futuro su desenvolvimiento dentro de su ambiente, la relación con pares, el aprendizaje de los currículos académicos y la posible presencia de problemas emocionales, en donde se ha demostrado que los niños con algún tipo de trastorno neuropsicológico presentan un índice más alto de trastornos emocionales y del comportamiento. 6 Dichos problemas pueden ser ocasionados durante el periodo perinatal y/o posnatal.7 Para el año 2010, el 21\% de las muertes de menores de un año en Colombia fueron ocasionados por trastornos específicos del periodo perinatal, razón por la cual es necesario indagar que ocurre con aquellos nacimientos que presentaron algún inconveniente y que en estos momentos se convierten en poblaciones en riesgo respecto a su desarrollo y desenvolvimiento en su ambiente escolar, familiar, emocional y social.8,9

Por su parte, las patologías neonatales como el nacimiento prematuro, dificultades respiratorias, hipoxia, asfixia, trauma obstétrico y bajo peso, son factores que influyen en la presencia de problemas en el neurodesarrollo. Según cifras del DANE, el bajo peso entre los recién nacidos en Colombia se ubica entre el $8 \%$ y $9 \%$ de los nacimientos; el DANE también afirma que en Boyacá, el 9\% de los mismos, presentan bajo peso. Por otro lado, un estudio llevado a cabo por la UNICEF estima que cada año, 20 millones de niños nacen prematuramente, lo que corresponde a un 14\% de la población mundial.10

Estas cifras dan cuenta de la situación, dado que los trastornos producidos antes del primer año de vida generan mayores alteraciones en edades posteriores, y se ha observado además, una relación directamente proporcional entre el CI bajo y dichos trastornos.11 Por otro lado, la mejora de condiciones asistenciales en el nacimiento conlleva a que se presenten poblaciones de riesgo debido al deficiente desarrollo del sistema nervioso del neonato y que en ocasiones pasan inadvertidas, hasta que la edad escolar demanda habilidades matemáticas, lectoras y escritoras.1,12

Dentro de la literatura consultada, se evidencia ausencia de estudios en cuanto a programas de intervención en Colombia sobre MN llevados a cabo con el fin de fortalecer las áreas de desarrollo global de la misma.

Las distintas investigaciones que se han realizado dentro del contexto latinoamericano y colombiano, enfatizan la evaluación de la MN con problemas específicos, la relación del peso y edad 
con $\mathrm{MN}$, las propiedades psicométricas de los instrumentos que evalúan $\mathrm{MN}$, más específicamente del CUMANIN, y su adaptación y validación a países como Perú y Colombia, o se centran en describir los índices de $\mathrm{MN}$ en muestras de preescolares.13-22

Por lo tanto, se plantea como objetivo general para esta investigación evaluar la madurez neuropsicológica en niños (as) de tres a seis años de un colegio privado de la ciudad de Tunja para elaborar a futuro un programa de corrección neuropsicológica en base a los resultados obtenidos. Al respecto, en Colombia la Ley 115 de 1994, establece dos tipos de instituciones educativas: las públicas que son brindadas por el gobierno en cuanto a la calidad y cubrimiento del servicio, y la privada que es ofrecida por personas particulares y que responde en esencia, en cuanto a parámetros y requerimientos, a las instituciones públicas.23

Sin embargo, diversos estudios evidencian la brecha existente entre instituciones públicas y privadas en cuanto a nivel educativo, características de los individuos y sus familias en relación a factores socioculturales, infraestructura y nivel educativo de los docentes favoreciendo a las instituciones privadas.24,25 Como objetivos específicos se propone evaluar el grado de madurez neuropsicológica en niños (as) preescolares y comparar los resultados por sexo y grupo etario.

\section{Materiales y métodos}

Se realiza un estudio observacional- descriptivo y comparativo de corte transversal, dado que se buscó identificar y cuantificar el nivel de desarrollo neuropsicológico de la muestra estudiada, detallando las características de la población en un momento determinado.26,27 Esto con el fin de identificar los problemas y necesidades existentes a partir de la aplicación del cuestionario de madurez neuropsicológica CUMANIN y una historia clínica, con el fin de diseñar un programa de corrección. La población del estudio estuvo constituida por los estudiantes de un colegio privado de la ciudad de Tunja, con edades comprendidas entre los 3 y 6 años. La muestra fue seleccionada no probabilísticamente y por conveniencia y consta de 46 estudiantes de los grados de pre jardín, jardín y transición, la cual se divide en 23 niños (50\%) y 23 niñas (50\%). Los criterios de inclusión fueron: (a) ser estudiante del colegio donde se realizó la investigación, (b) tener edades entre los 36 y 78 meses al momento de la investigación, (c) tener autorización por parte de sus padres y/o representante legal para la participación de este estudio a partir del consentimiento informado y (d) no presentar déficits visuales, motores, auditivos o cognitivos que impidieran o dificultaran el desarrollo de la prueba.

Como instrumento se empleó la Historia Clínica Infantil que es un cuestionario diseñado para los padres de los niños, en donde se recoge información mediante preguntas abiertas y cerradas sobre la historia evolutiva a nivel prenatal, las condiciones gestacionales, los inconvenientes $\mathrm{y}$ enfermedades durante el embarazo, el consumo de sustancias y de alcohol. También tiene en cuenta la historia médica del participante, su estado de salud actual, las enfermedades que ha tenido a lo largo del ciclo vital y antecedentes familiares. El desarrollo neuromuscular, el habla, los hábitos la conducta, el juego, la historia educativa, relación de los padres y hermanos y antecedentes psicológicos familiares.

También se empleó el Cuestionario de Madurez Neuropsicológica CUMANIN, 1 en niños y niñas con edades entre los 36 y 78 meses, su aplicación es individual con un tiempo entre 30 a 50 minutos. Está compuesta por 83 ítems contenidos en 8 escalas principales y 5 auxiliares con un total de 13 sub escalas, su puntuación es entre 0 (error) y 1 (acierto). Presenta una subescala de lateralidad. Las ocho subescalas principales son: psicomotricidad, lenguaje articulatorio, lenguaje comprensivo, lenguaje expresivo, estructuración espacial, viso percepción, memoria y ritmo. Dichas áreas han sido determinadas a partir del consenso de la comunidad científica; el instrumento arroja un coeficiente de desarrollo (CD) un desarrollo verbal (DV) y un desarrollo no verbal (DNV).28 A su vez, se describe que este 
instrumento es válido y su consistencia interna oscila entre 0,71 y 0,92 en sus diferentes subescalas. Para la evaluación se usó la adaptación semántica para el contexto colombiano realizada por Ávila,16 donde se evidencian índices de confiabilidad y validez similares a la prueba original.

La ejecución del proyecto siguió estas fases:

Primera fase: El proyecto fue presentado, evaluado y aprobado bajo la convocatoria de sostenibilidad de grupos de investigación de la Dirección de Investigaciones de la UPTC.

Segunda fase: Contacto con el colegio para la evaluación: Se realiza contacto con la institución con el fin de presentar y aprobar el proyecto.

Tercera fase: Se realizó una reunión con los padres de los participantes a quienes se les describió el proyecto y se les entregó el consentimiento informado. Se presentó el objetivo de la investigación, el procedimiento de la investigación que contempla la aplicación de la historia clínica y el CUMANIN; el diseño del programa de corrección neuropsicológica de acuerdo con los resultados de la evaluación de la madurez neuropsicológica y la futura aplicación del mismo una vez terminado, la posibilidad de no participar en la investigación, los posibles riesgos, normas éticas y derecho a recibir resultados obtenidos con cada uno de los participantes si este los solicita, lo que se espera obtener y los beneficios de la investigación.

Cuarta fase: Se administra el cuestionario de Madurez Neuropsicológica CUMANIN. La aplicación fue individual y se realizó en las oficinas de piscología y/o coordinación que cumplieran los requisitos ambientales de aplicación. A su vez, los padres de los participantes registraron y entregaron la historia clínica a los investigadores.

Quinta fase: Se elabora la base de datos mediante el software SPSSS, teniendo en cuenta los resultados y calificaciones de los 46 participantes con el fin de obtener el índice de desarrollo de la muestra estudiada. Se identificaron las características seleccionadas para esta investigación.

Sexta fase: A los resultados obtenidos se les dio un tratamiento estadístico de cálculo de medias y desviaciones a partir del valor promedio de la prueba adaptada al contexto colombiano, cálculo de frecuencias según los niveles de desempeño (por debajo, dentro y por encima de la media) y la comparación de resultados entre grupos de edad y sexo. Se obtuvieron los resultados demandados por esta investigación y se realizó el informe final del presente proyecto. Además se hizo entrega de los resultados individualmente a los padres de familia.

Para el análisis estadístico, se realiza una descripción del contexto sociocultural de la muestra a partir de los datos obtenidos en la historia clínica mediante el cálculo de frecuencias, se hizo un cálculo de la consistencia interna de los resultados de cada una de las sub escalas del CUMANIN por medio del estadístico alfa de Cronbach. Luego de esto, se realizó un análisis de la normalidad de los datos mediante la prueba de normalidad de Kolmogorov-Smirnov con el fin de determinar los resultados de la estadística descriptiva teniendo en cuenta las puntuaciones por grupo de edad y la frecuencia de respuestas según el rendimiento neuropsicológico (bajo, dentro de la media y por encima de la media). Además, se describió el desempeño de la muestra teniendo en cuenta los grupos de edad y la frecuencia entre las puntuaciones. Por último, se realizó una comparación de resultados entre sexos por medio del estadístico T-Student.

\section{Consideraciones Éticas}

Este proyecto de investigación se califica como investigación con riesgo mínimo, ya que involucra el registro de datos a través de procedimientos comunes, según la clasificación establecida en la Resolución 8430 de 1993 del Ministerio de Salud. La elaboración del consentimiento informado cumple con todos los lineamientos éticos establecidos en la Ley 1090 de 2006 de participación voluntaria, confidencialidad en los datos empleados, así como la información completa acerca de lo que se va a realizar, los riesgos, los instrumentos utilizados y los resultados esperados. 


\section{Resultados}

Los datos de antecedentes personales, familiares y hábitos se pueden observar en la Tabla 1. El 59\% de los participantes tienen hermanos y el $41 \%$ son hijos (as) únicos (as). La muestra estudiada es tomada de un colegio de carácter privado de la ciudad de Tunja, que tiene características particulares en cuanto a su desarrollo social y cultural. Dentro de los datos obtenidos por medio de la historia clínica de los participantes, corroboradas en entrevista con los padres, se evidencia que los padres de la comunidad educativa se desenvuelven en estratos sociales medios y altos teniendo residencia en zona urbana de la ciudad de Tunja.

Por su parte el $75,7 \%$ de los padres de los niños evaluados reportan vivir en pareja y el $24,3 \%$ son separados o madres solteras. En cuanto a la escolaridad de los padres, los resultados evidencian que los papas tienen escolaridad técnico, universitaria o superior, mientas que las madres tienen nivel educativo más alto que su pareja. (Tabla 1).

Tabla 1. Caracterización de la muestra

\begin{tabular}{|c|c|c|c|}
\hline \multicolumn{4}{|l|}{ Antecedentes personales } \\
\hline Peso al nacer & $\begin{array}{c}\text { Bajo } \\
5 \%(2)\end{array}$ & \multicolumn{2}{|c|}{$\begin{array}{l}\text { Normal } \\
95 \%(44)\end{array}$} \\
\hline Desarrollo del lenguaje & $\begin{array}{l}\text { Dificultad } \\
22 \%(10)\end{array}$ & \multicolumn{2}{|c|}{$\begin{array}{c}\text { Normal } \\
78 \%(36)\end{array}$} \\
\hline Desarrollo motor & $\begin{array}{l}\text { Dificultad } \\
7 \%(3)\end{array}$ & \multicolumn{2}{|c|}{$\begin{array}{l}\text { Normal } \\
93 \%(43)\end{array}$} \\
\hline Lateralidad & $\begin{array}{c}\text { Cruzada (do) } \\
67 \%\end{array}$ & \multicolumn{2}{|c|}{$\begin{array}{c}\text { Consistente } \\
33 \%\end{array}$} \\
\hline & Diestro (a) & $\begin{array}{l}\text { Zurdo } \\
\text { (a) }\end{array}$ & $\begin{array}{l}\text { Mixto } \\
\text { (a) }\end{array}$ \\
\hline Mano & $86 \%(30)$ & $\begin{array}{c}14 \% \\
(6)\end{array}$ & $\begin{array}{l}0 \% \\
(0)\end{array}$ \\
\hline Pie & $65 \%(30)$ & $\begin{array}{l}21 \% \\
(10)\end{array}$ & $\begin{array}{c}14 \% \\
(6)\end{array}$ \\
\hline Ojo & $47 \%(22)$ & $\begin{array}{l}47 \% \\
(22)\end{array}$ & $\begin{array}{l}6 \% \\
(2)\end{array}$ \\
\hline $\begin{array}{l}\text { Enfermedad médica } \\
\text { actual }\end{array}$ & $\begin{array}{c}\mathrm{Si} \\
22 \%(22)\end{array}$ & \multicolumn{2}{|c|}{$\begin{array}{c}\text { No } \\
78 \%(36)\end{array}$} \\
\hline Escolarización & $\begin{array}{l}1 \text { a } 2 \text { años } \\
59 \% \text { (27) }\end{array}$ & $\begin{array}{l}3 \mathrm{a} \\
41 \%\end{array}$ & \\
\hline
\end{tabular}

\begin{tabular}{lcc}
\hline Antecedentes familiares & & \\
\hline Hermanos & $\begin{array}{c}\text { Hijo (a) único } \\
(\mathrm{a})\end{array}$ & Tiene hermanos \\
& $41 \%(19)$ & $59 \%(27)$ \\
Escolaridad del padre & Tec. & $\begin{array}{c}\text { Bachiller o } \\
\text { inferior }\end{array}$ \\
& $\begin{array}{c}\text { universitario o } \\
\text { superior } \\
\text { 81\% (37) }\end{array}$ & $19 \%(9)$ \\
$\begin{array}{l}\text { Escolaridad de la } \\
\text { madre }\end{array}$ & Tec. & Bachiller o \\
& inferior \\
Anersitaria o & superior & $10 \%(5)$ \\
Antecedentes & $90 \%(41)$ & No \\
y/o neurológicos & Si & $88 \%(40)$ \\
Hábitos & $12 \%(6)$ & \\
Sueño & & Normal \\
& Dificultad & $85 \%(30)$ \\
Alimentación & $15 \%(6)$ & Normal \\
& Dificultad & $90 \%(41)$ \\
\hline
\end{tabular}

Tabla 2. Distribución de los participantes por grupo de edad, sexo y lateralidad

\begin{tabular}{lrrrr}
\hline \multirow{2}{*}{$\begin{array}{l}\text { Grupo de edad } \\
\text { en meses }\end{array}$} & \multicolumn{5}{c}{ Masculino } & \multicolumn{3}{c}{ Femenino } \\
& N & \% & N & \% \\
\hline $36-42$ & 6 & 26 & 0 & 0 \\
$43-48$ & 3 & 13 & 0 & 0 \\
$49-54$ & 6 & 26 & 2 & 8.8 \\
$55-60$ & 3 & 13 & 6 & 26 \\
$61-66$ & 1 & 4,4 & 10 & 4,4 \\
$67-72$ & 4 & 17,4 & 4 & 17,4 \\
$73-78$ & 0 & 0 & 1 & 4,4 \\
Total & 23 & 100 & 23 & 100 \\
Lateralidad & & & & \\
Consistente & 6 & 26 & 9 & 39.1 \\
Cruzado & 17 & 74 & 14 & \\
Total & 23 & 100 & 23 & 100 \\
\hline
\end{tabular}

La muestra estudiada presenta una media de edad de 57 meses con distribución similar entre el sexo de los participantes. Con lateralidad consistente del total de niños y niñas (15) se encontró el $32,6 \%$ y el $67,4 \%$ restante (31), tienen lateralidad cruzada. La función del sexo se observa que la lateralidad consistente es más frecuentes en niñas $(39,1)$ en tanto que la cruzada lo es en niños 
(74\%). El análisis de confiabilidad muestra consistencia interna en las sub escalas. Específicamente, la sub escala de memoria icónica muestra un coeficiente alfa denominado como 'inaceptable'.29 Aun así, las otras sub escalas indican que en general, los resultados obtenidos son aceptables. La prueba de KolmogorovSmirnov muestra una distribución normal entre las subescalas de la prueba, el desarrollo normal y el cociente de desarrollo evaluado en el CUMANIN, teniendo en todos los resultados valores mayores a $p=0.05$. El análisis de normalidad permite hablar de medias y desviaciones, puesto que la distribución de los resultados en las diferentes escalas y sub escalas en la población estudiada es normal. (Tabla 3).

Tabla 3. Confiabilidad y normalidad de la prueba en la muestra estudiada

\begin{tabular}{|c|c|c|c|c|c|c|c|}
\hline Escala & $\begin{array}{l}\alpha \text { Muestra } \\
\text { estudiada }\end{array}$ & No. elementos & $\alpha$ Original & $\begin{array}{l}\text { Kolmogorov- } \\
\text { Smirnov }\end{array}$ & $\begin{array}{c}\text { Sig. } \\
\text { Asintótica }\end{array}$ & $\mathbf{M}$ & DE \\
\hline Psicomotricidad & 0,49 & 11 & 0,71 & 1,36 & 0,050 & 7,78 & 1,77 \\
\hline L. Articulatorio & 0,81 & 15 & 0,92 & 1,02 & 0,255 & 9,63 & 3,59 \\
\hline L. Expresivo & 0,61 & 4 & 0,73 & 1,30 & 0,069 & 2,37 & 1,29 \\
\hline L. Comprensivo & 0,7 & 9 & 0,72 & 0,89 & 0,395 & 3,74 & 2,19 \\
\hline Estr. Espacial & 0,7 & 12 & 0,81 & 0,87 & 0,439 & 9,67 & 2,82 \\
\hline Visopercepción & 0,9 & 15 & 0,91 & 0,84 & 0,487 & 7,15 & 4,37 \\
\hline Memoria & $-0,12$ & 10 & 0,57 & 1,17 & 0,131 & 6,93 & 1,31 \\
\hline Ritmo & 0,51 & 7 & 0,72 & 1,15 & 0,143 & 2,72 & 1,32 \\
\hline Desarrollo verbal & & & & 0,77 & 0,586 & 15,76 & 5,51 \\
\hline Desarrollo no verbal & & & & 0,95 & 0,333 & 34,30 & 9,02 \\
\hline Cociente de desarrollo & & & & 0,52 & 0,953 & 101,85 & 10,95 \\
\hline
\end{tabular}

El análisis estadístico descriptivo indica que el valor del cociente de desarrollo perfila a los niños (as) de este estudio dentro de la media poblacional, ya que presentan un resultado de 101,85 del coeficiente de desarrollo, teniendo en cuenta que la media estimada es 100 permitiendo una desviación estándar de 10 puntos por encima y 10 puntos por debajo. La puntuación total de esta prueba teniendo en cuenta el rango de edad se muestra en el siguiente gráfico:

Grafico 1. Distribución del total de las puntuaciones directas por edad

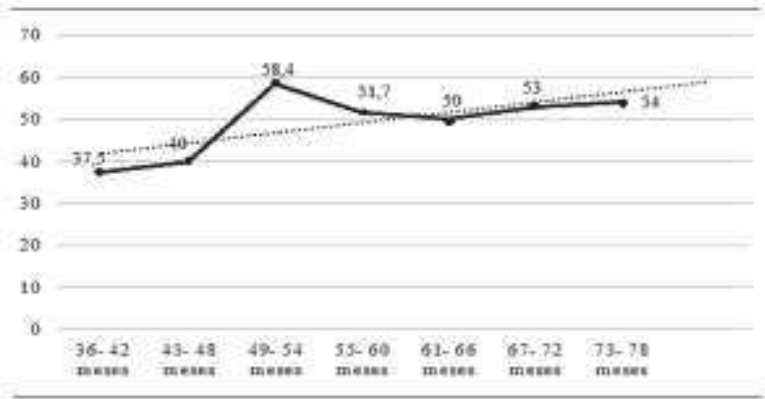

La línea de ajuste muestra una tendencia leve de crecimiento, lo cual indica que la prueba actúa como una prueba de desarrollo neuropsicológico, puesto que a mayor edad, mayor crecimiento en las puntuaciones. Por otra parte, se observa que el desempeño total del CUMANIN en la edad comprendida entre 49- 54 meses es superior a las siguientes edades.

\section{Gráfico 2. Puntuaciones de las subescalas del CUMANIN por grupos de edad}

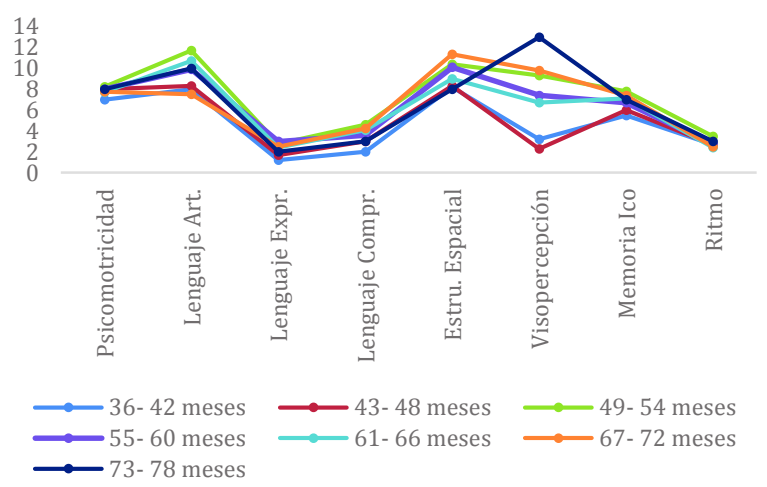


Con respecto a la ejecución en cada una de las sub escalas, (Gráfico 2) los resultados no presentan la distribución esperada suponiendo que a mayor edad, mejor ejecución en cada una de las sub escalas. Se evidencia que el desempeño general de la edad comprendida entre 55-60 meses, 61-66 meses, 67-72 meses y 73-78 meses es inferior a la edad de 49-54 meses, que presenta un resultado esperado teniendo en cuenta la prueba original. Las sub escalas de estructuración espacial y viso percepción son las que más presentan resultados pobres conforme aumenta la edad de los niños.

La frecuencia de puntuaciones, teniendo en cuenta el grupo de edad y el sexo se describirán a continuación teniendo en cuenta los centiles de la prueba y cuyos resultados indican que el centil de 5 a 39 se tipifican como bajo rendimiento neuropsicológico y se debe tener un seguimiento y/o una intervención; centil 40 a $60=$ entre la media poblacional; centil 61 a $99=$ por encima de la media poblacional.1

Tabla 4. Frecuencia de puntuaciones por escala

\begin{tabular}{|c|c|c|c|}
\hline Escala & $\begin{array}{c}\text { Centil } 5 \text { a } \\
39\end{array}$ & $\begin{array}{c}\text { Centil } 40 \text { a } \\
60\end{array}$ & Centil 61 a 99 \\
\hline Psicomotricidad & $9(19,6 \%)$ & $12(26,1 \%)$ & $25(64,3 \%)$ \\
\hline $\begin{array}{l}\text { Lenguaje } \\
\text { articulatorio }\end{array}$ & $\begin{array}{r}14 \\
(30,4 \%)\end{array}$ & $24(52,2 \%)$ & $8(17,4 \%)$ \\
\hline $\begin{array}{l}\text { Lenguaje } \\
\text { expresivo }\end{array}$ & $\begin{array}{r}18 \\
(39,1 \%)\end{array}$ & $10(21,8 \%)$ & $18(39,1 \%)$ \\
\hline $\begin{array}{l}\text { Lenguaje } \\
\text { comprensivo }\end{array}$ & $\begin{array}{r}16 \\
(34,8 \%)\end{array}$ & $15(32,6 \%)$ & $15(32,6 \%)$ \\
\hline $\begin{array}{l}\text { Estructuración } \\
\text { espacial }\end{array}$ & $6(13 \%)$ & $9(19,6 \%)$ & $31(67,4 \%)$ \\
\hline Visopercepción & $\begin{array}{r}11 \\
(23,9 \%)\end{array}$ & $13(28,6 \%)$ & $22(47,5 \%)$ \\
\hline Memoria icónica & $3(6,6 \%)$ & $7(15.4 \%)$ & $36(78 \%)$ \\
\hline Ritmo & & $\begin{array}{r}10 \\
(21,8 \%)\end{array}$ & $\begin{array}{r}29 \\
(62,8 \%)\end{array}$ \\
\hline
\end{tabular}

La frecuencia de resultados por debajo de la media poblacional, es decir, con bajo rendimiento neuropsicológico y que requieren de un seguimiento y/o intervención, fueron los siguientes: en la escala de psicomotricidad, el
20\% (9 niños) de la población estudiada, en lenguaje articulatorio, un 30\% (14 niños) de la población. El 13\% (6 niños) se encuentra en el centil 5 a 39 en la escala de estructuración espacial; el 39\% presenta bajo rendimiento en la escala de lenguaje expresivo (18 niños). En lenguaje comprensivo el 35\% 16 niños), en visopercepción el 24\% (11 niños), en memoria icónica $6 \%$ (niños) y por último, $22 \%$ en la escala de ritmo (10 niños).

Además, el 63\% (29 niños) de la población estudiada, presenta un puntaje por debajo de las esperadas en por lo menos dos de las áreas evaluadas, mientras que solo el 6\% (3 niños) de la población obtuvo puntuaciones acordes con su edad de desarrollo. La tabla que se muestra a continuación indica las diferencias entre los dos sexos realizados a partir del análisis estadístico TStudent:

Tabla 5. Diferencia del desempeño entre sexos

\begin{tabular}{|c|c|c|c|c|c|c|}
\hline & \multicolumn{2}{|c|}{ Masculino } & \multicolumn{2}{|c|}{ Femenino } & \multicolumn{2}{|c|}{$\begin{array}{c}\text { Diferencias entre } \\
\text { sexos }\end{array}$} \\
\hline & $\mathbf{M}$ & DE & $\mathbf{M}$ & DE & $\begin{array}{c}\text { T- } \\
\text { Student }\end{array}$ & $\begin{array}{c}\text { Significan } \\
\text { cia }\end{array}$ \\
\hline $\begin{array}{l}\text { Psicomotrici } \\
\text { dad }\end{array}$ & 7,61 & 1,99 & 7,96 & 1,55 & 3,25 & 0,078 \\
\hline $\begin{array}{l}\text { Lenguaje } \\
\text { articulatorio }\end{array}$ & 9,78 & 3,71 & 9,48 & 3,55 & 0,03 & 0,861 \\
\hline $\begin{array}{l}\text { Lenguaje } \\
\text { expresivo }\end{array}$ & 2,04 & 1,55 & 2,70 & 0,88 & 11,72 & 0,001 \\
\hline $\begin{array}{l}\text { Lenguaje } \\
\text { comprensivo }\end{array}$ & 3,65 & 2,19 & 3,83 & 2,23 & 0,07 & 0,787 \\
\hline $\begin{array}{l}\text { Estructuraci } \\
\text { ón espacial }\end{array}$ & 9.91 & 3,52 & 9,43 & 1,95 & 14,11 & 0,001 \\
\hline $\begin{array}{l}\text { Visopercepci } \\
\text { ón }\end{array}$ & 6.96 & 4,76 & 7,35 & 4,03 & 0,58 & 0,451 \\
\hline $\begin{array}{l}\text { Memoria } \\
\text { icónica }\end{array}$ & 6,70 & 1,33 & 7,17 & 1,27 & 0,10 & 0,749 \\
\hline Ritmo & 2,87 & 1,29 & 2,57 & 1,44 & 0,50 & 0,483 \\
\hline $\begin{array}{l}\text { Fluidez } \\
\text { verbal }\end{array}$ & $\begin{array}{r}42,3 \\
5\end{array}$ & $\begin{array}{r}42,3 \\
2\end{array}$ & $\begin{array}{r}56,4 \\
3\end{array}$ & $\begin{array}{r}40,9 \\
4\end{array}$ & 0,05 & 0,820 \\
\hline Atención & 7,74 & 3,81 & 7,87 & 2,93 & 1,66 & 0,204 \\
\hline $\begin{array}{l}\text { Desarrollo } \\
\text { verbal }\end{array}$ & $\begin{array}{r}15,3 \\
9\end{array}$ & 6,19 & $\begin{array}{r}16,1 \\
3\end{array}$ & 4,87 & 2,16 & 0,148 \\
\hline $\begin{array}{l}\text { Desarrollo } \\
\text { no verbal }\end{array}$ & $\begin{array}{r}34,1 \\
3\end{array}$ & $\begin{array}{r}10,5 \\
5\end{array}$ & $\begin{array}{r}34,4 \\
8\end{array}$ & 7,43 & 2,78 & 0,102 \\
\hline $\begin{array}{l}\text { Cociente } \\
\text { desarrollo }\end{array}$ & $\begin{array}{r}101 \\
83\end{array}$ & $\begin{array}{r}11,7 \\
6\end{array}$ & $\begin{array}{r}101 \\
87\end{array}$ & $\begin{array}{r}10,3 \\
5\end{array}$ & 0,89 & 0,350 \\
\hline
\end{tabular}

La tabla 5 muestra la comparación de los resultados obtenidos entre los dos sexos, teniendo en cuenta que se dividían similarmente 
entre 23 niños y 23 niñas. Los resultados muestran que no existen diferencias significativas en la ejecución entre sexos. Los datos obtenidos en la prueba T- Student evidencian una significancia bilateral menor a 0,05 en dichas sub escalas, que indica diferencias entre los dos sexos en las áreas de lenguaje expresivo y estructuración espacial. Por su parte, el resto de sub escalas, el desarrollo verbal, no verbal y el coeficiente de desarrollo no muestran diferencias significativas entre los dos sexos.

\section{Discusión}

Como primera instancia en la evaluación del desarrollo neuropsicológico, se encuentran puntuaciones dentro de lo esperado para la edad y la escolaridad de la muestra (Gráfico 1) indicando que a mayor edad, mejores resultados en las puntuaciones. Estos resultados son similares en los encontrados en Ávila, ${ }_{16}$ Campo, et al.,15 Portellano et al.,4 Uribe,18 Urzúa et al.,19 Los resultados para cada una de las subescalas evidencian una mejor ejecución por parte del grupo de edad de 49 a 54 meses respecto a los demás grupos etarios en la mayoría de las sub escalas (Gráfico 2); los resultados del grupo de edad referido, coinciden a lo reportado en la prueba original, los que indican que la prueba responde a la evaluación del desarrollo neuropsicológico.1

Sin embargo, los grupos de mayor edad deberían suponer puntuaciones superiores al grupo de 49 a 54 meses, entendiendo que los procesos de evolución funcional que ocurren en el cerebro en esta edad, junto a los procesos de maduración, de crecimiento axonal y dendrítico, la formación de nuevas sinapsis y el desarrollo de la mielinización, deberían ser más rápidos y por consiguiente, presentar mejores resultados conforme aumenta la edad.3,30,31 Se debe tener en cuenta que el número de participantes para cada grupo de edad no era homogéneo, por tanto estos resultados deben ser tomados con precaución y con fines descriptivos para la muestra estudiada.

Adicionalmente, se encuentra que la frecuencia de resultados con bajo rendimiento neuropsicológico (por debajo del centil 40) se observan en todas y cada una de las subescalas. En referencia, el 63\% de los participantes tienen puntuaciones por debajo de las media en al menos dos de las áreas evaluadas y solo el 6\% (3 participantes) de toda la muestra, reportan puntuaciones acordes a su edad de desarrollo. Estudios anteriores registran resultados similares en algunas sub escalas, respecto a la frecuencia de poblaciones con centiles por debajo de 45.15,20,22 Estas coincidencias son tomadas para la corrección de los perfiles de los niños ya que presentar puntuaciones por debajo de las admitidas y pueden ser un indicador de poblaciones en riesgo de fracaso escolar.13,14,36

Se debe tener en cuenta que, "aunque las puntuaciones bajas en el CUMANIN no predeterminan necesariamente cuál va a ser el pronóstico del rendimiento cognitivo de un niño, hay que entender que un deficiente nivel de rendimiento en esta prueba es un signo de alerta que siempre debe tenerse en cuenta como factor de riesgo".4

Los estudios revisados, realizan las investigaciones en poblaciones de otras características socioeconómicas (hogares de paso y de estrato socioeconómico bajo), que son diferentes a las de esta investigación, la cual se desarrolla en un colegio privado, situación que en la literatura reporta que la relación entre el tipo de institución a la que asisten los escolares son determinantes en la madurez neuropsicológica, favoreciendo a la institución privada debido a factores económicos y educacionales de los padres.13,15,19-22,32-34

Cada uno de los resultados tienen un significado neuro- funcional para cada escala, es así como puntuaciones bajas en de psicomotricidad, indican el nivel de desarrollo en las estructuras encefálicas como el procesamiento en las áreas parietales, cerebelosas, ganglios basales, tálamo y la corteza premotora, lo cual se interpreta según los criterios de calificación del CUMANIN, que el $20 \%$ de los niños (as) presentarían inmadurez en esta función.4,8 La no intervención sobre psicomotricidad, podría tener a futuro dificultades psicomotoras desde dispraxia del desarrollo hasta hiperactividad. Esta función 
psicomotora es necesaria y está vinculada con la adquisición de habilidades escolares, deportivas, laborales y artísticas.35,36

Las áreas de lenguaje evaluadas son las que presentan puntajes más bajos dentro del perfil neuropsicológico con un porcentaje de 30\%, 39\% y $35 \%$ de la muestra en las diferentes edades evaluadas, que se ubica por debajo de la media poblacional en las áreas de lenguaje articulatorio, expresivo y comprensivo respectivamente, y que a su vez, involucran estructuras anatómicas a las cuales se debe tener en consideración como el lóbulo frontal, temporal, el área de broca y el área de Wernicke.8,37,38

El $13 \%$ de la muestra estudiada presenta puntuaciones por debajo de la media en el área de estructuración espacial relacionada con las áreas asociativas de la corteza parieto- temporooccipital y las áreas de asociación del lóbulo occipital, se ven estrechamente relacionadas con las funciones viso perceptivas, indicando inmadurez neuropsicológica en el $24 \%$ de los participantes que presentan puntuaciones muy por debajo de la media en esta escala.4,39 El área de visopercepción se relaciona con el funcionamiento de áreas visuales secundarias y asociativas del lóbulo occipital, las vías de asociación cortical, las áreas de asociación de la corteza cerebral sensorial.18,31,40 El 24\% de los participantes presentan inmadurez en dichas estructuras.

La prueba de memoria icónica, según Portellano y

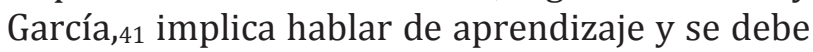
tener en cuenta aspectos como la memoria operativa relacionada con estructuras como la corteza parietal, hipocampo y amígdala. Así, la ejecución en las tareas de memoria icónica implica la activación de dichas estructuras además de la utilización del hemisferio derecho.37 El $6 \%$ de la muestra estudiada presenta puntuaciones deficitarias en estas tareas, que lo relaciona directamente con dicho hemisferio.

Respecto a la última subescala evaluada- ritmo-, la literatura refiere la importancia de los procesos rítmicos en el desarrollo motor, la adquisición de habilidades de escritura, musicales, lenguaje y audición.3,31,42 Dada la importancia de este proceso, se debe tener un seguimiento sobre el $22 \%$ de los participantes que se ubicaron por debajo del centil 40 y que los identifica como población que demanda alguna medida correctiva, implicando áreas estructurales del lóbulo temporal derecho y el sistema reticular activador ascendente.4

Al comparar la ejecución de los sexos, Ávila,16 refiere una ligera ventaja de las niñas en las áreas del lenguaje, mientras que los niños presentan mejor desempeño en el área espacial, coincidiendo con los resultados de esta investigación, donde se identificó diferencias estadísticamente significativas en el desempeño de las niñas en la sub escala de lenguaje expresivo y de los niños en estructuración espacial (Tabla 4). Estos hallazgos, ya se han descrito anteriormente en la literatura científica.30,43-46 Respecto a las demás subescalas, no se identificaron diferencias significativas entre los sexos, coincidiendo con hallazgos previos.4,19,34

\section{Conclusiones}

Los datos reportados permiten evidenciar la conveniencia del diseño de un programa de corrección neuropsicológica para la muestra estudiada, puesto que al encontrar puntuaciones por debajo de la media en por lo menos dos subescalas del CUMANIN, "se sugiere arbitrar medidas correctivas para potenciar la función decaída, favorecida por la plasticidad cerebral infantil". 3 Por su parte, el diseño del programa adquiere mayor relevancia debido a la fase evolutiva en la que se encuentran los niños en el momento de la intervención, donde se espera obtener un mejor pronóstico a comparación de intervenciones tardías. 16,47

Los perfiles que se presentan en el apartado de los resultados, demuestran que el $63 \%$ de los niños presentan puntuaciones deficitarias en por lo menos dos escalas, lo que justifica que se diseñe un programa de corrección neuropsicológica, tomando como línea base las funciones de su desempeño en el CUMANIN, como lo recomienda el autor. No existen publicaciones que obedezcan a las sugerencias de Portellano et al.4 Los 
resultados de este estudio no muestran perfiles deteriorados a nivel general, evidenciando patología (tres o más escalas por debajo del centil 40) y por tanto, no es necesario que el diseño del programa sea específico e individual como se sugiere se realice en los casos de rehabilitación neuropsicológica y la aplicación de medidas de reentrenamiento, compensación y/o sustitución.41,48

Además, la implementación de un programa de prevención dirigido únicamente a la aplicación individual, acarrearía costos y tiempo insuficientes para su aplicación en población no patológica.49 El programa se diseña de forma lúdica para que resulta agradable con los niños beneficiando el juego temático, juego de roles, juegos con reglas y juegos creativos, los cuales son denominados como actividades rectoras de la edad preescolar, que benefician la adquisición de normas y relaciones sociales, el desarrollo del pensamiento reflexivo, la regulación de la conducta y el desarrollo de la imaginación, siendo esta interacción, la base de preparación para la actividad escolar.36,50,51

Las fortalezas de esta investigación se enmarcan en el acceso a la muestra estudiada, el gran entusiasmo por parte de los padres de familia en la ejecución del proyecto. Por último, estos resultados hacen parte de la primera fase de la investigación, donde se espera ir más allá de la descripción de la madurez neuropsicológica, diseñando e implementando a futuro el programa de corrección neuropsicológica.

\section{Recomendaciones}

El diseño del programa de corrección y fortalecimiento de las áreas globales del desarrollo estudiadas se deben dar a partir de la creación de ejercicios dirigidos a mejorar dichas funciones mentales. Además, debe tener en cuenta el nivel de madurez que ya se tienen previamente consolidas en los niños para cada grupo de edad, zonas de desarrollo próximo y procesos neuromadurativos demandados en cada una de las actividades. A su vez, se deben realizar sesiones lúdicas, creativas y con la implementación de refuerzos positivos tanto materiales como sociales en las diferentes actividades, y por último, tener en cuenta el grupo de edad, optando por diferentes sesiones para cada grupo de edad (tres- cuatro años) (cinco-seis años) teniendo en cuenta las instrucciones y dificultad de cada una de las actividades, debido a que las estructuras anatómicas encargadas en la ejecución de las funciones mentales están más desarrolladas en los niños de cinco y seis años, respecto a los menores.

Financiación: Este trabajo es parte de los productos de un proyecto de investigación aprobado y financiado por la Dirección de Investigaciones de la Universidad Pedagógica y Tecnológica de Colombia. Código SGI- 1589.

Conflicto de intereses. Ninguno declarado por los autores.

\section{Referencias}

1. Benítez Y. Signos neurológicos menores en la edad preescolar. Revista Mexicana de Neurociencia. 2008;9(6):445- 453.

2. Encalada V, Reino M. Evaluación de la madurez neuropsicológica de los niños y niñas de nivel inicial. [tesis de maestría]. Universidad de Cuenca. Cuenca, Ecuador. 2013.

3. Guerrero M, Guillen D, Sato L. Desarrollo neuropsicológico en niños preescolares con exposición crónica a plomo, residentes en el Callao - Perú. Revista. Pediatría. 2011;64(2):9-15.

4. Portellano J, Mateos R, Martínez R. Cuestionario de Madurez Neuropsicológica infantil CUMANIN. Madrid, España: TEA Ediciones. 2000.

5. Chávez R. Neurodesarrollo neonatal infantil. Un enfoque multi- inter y trans disciplinario e la prevención del daño. México DF, México: Editorial Médica Panamericana. 2003.

6. Semrud-Clikeman M, Ellison A. Neuropsicología infantil. Evaluación e intervención en los trastornos neuroevolutivos. (2ํ․ Ed.). Madrid, España: Pearson Ediciones. 2011.

7. Dzib A. Alteraciones del desarrollo por dificultades perinatales y la confusión con los trastornos del espectro autista. Revista Mexicana de neuropsicología.2010; 5(1), 4-9.

8. Belfort M, Santo E, McCormick M. Using parent questionnaries to assess neurodevelopment in former preterm infants: a validation study. Pediatric and Perinatal Epidemiology. 2012. 27:199- 207.

9. Colombia. Ministerio de Salud y Protección Social. Análisis de situación de salud según regiones Colombia. Informe de la Dirección de Epidemiologia y Demografía. Grupo ASIS. 2013.

10. Orozco C. Estado de la salud neonatal. 2012. (Formato HTML). Disponible en: 
http://aprendeenlinea.udea.edu.co/lms/moodle/mod /resource/view.php?inpopup=true\&id=102951

11. Junqué C, Barroso J. Manual de neuropsicología. Madrid, España: Editorial Síntesis. 2009.

12. Robaina G, Ruíz Y, Domínguez F, Roca C, Riesgo S, Berdayes J. Neurodesarrollo en recién nacidos ventilados con menos de 1.500 gramos. Revista Cubana de Pediatría. 2000;72 (4): 267- 274.

13. Barba F, Robles M. Utilidad del cuestionario CUMANIN para detectar diferencias, en dos grupos de preescolares, dentro de un programa de atención primaria. Electronic Journal of Research in Educational Psychology. 2012; 10 (26), 311- 332.

14. Vilatuña M. Evaluación neuropsicológica en niños de cuatro a cinco años con problemas de lenguaje. Quito. [Tesis de maestría]. Universidad Central del Ecuador. 2012.

15. Campo C, Tuesca R, Campo L. Relación entre el grado de madurez neuropsicológica infantil y el índice de talla y peso en niños de 3 a 7 años escolarizados de estratos socioeconómicos dos y tres de la ciudad de Barranquilla (Colombia). Salud Uninorte. Barranquilla. 2012;28(1):88-98.

16. Ávila A. Adaptación del cuestionario de madurez neuropsicológica de Portellano. Revista Iberoamericana de Psicología: Ciencia y Tecnología. 2012;5(1):91- 99.

17. Guerrero M. Adaptación del cuestionario de madurez neuropsicológica infantil CUMANIN - en una población urbana de Lima. Revista Electrónica del Instituto Psicología y Desarrollo. 2006; 8:1- 14.

18. Uribe M. Adaptation and standardization of Cuestionario para Madurez Neurológica Infantil (CUMANIN) at the State of Mexico. Honolulu. [Tesis Doctoral]. Atlantic International University. 2009.

19. Urzúa A, Ramos M, Alday C, Alquinta A. Madurez neuropsicológica en preescolares: propiedades psicométricas del test CUMANIN. Terapia psicológica. 2009;28(1):13-25.

20. Alonso A. De Luca C, Hutchinson E, Spencer M, Roberts G, Doyle L. Madurez neuropsicológica en niños de nivel inicial [tesis de maestría]. Developmental Neuropsychology, 2011;36(1):57- 73.

21. Campo L. Características del desarrollo cognitivo y del lenguaje en niños de edad preescolar. Psicogente. 2009;12(22):341-351.

22. Tarazona O. Evaluación neuropsicológica en niños pertenecientes a hogares infantiles del Bienestar Familiar. Regional Pamplona. Bucaramanga. [Tesis de Especialización]. Universidad Pontificia Bolivariana. 2008.

23. Colombia. Congreso de la Republica. Ley 115/ 1994 de 8 de Febrero, Ley General de Educación.

24. Núñez J, Steiner R, Cadena X, Pardo R. ¿Cuáles colegios ofrecen mejor educación en Colombia? Archivos de Economía. 2002;193: 1-56.

25. Iregui A, Melo L, Ramos J. Análisis de eficiencia de la educación en Colombia. Revista de Economía del Rosario. 2007;10(1):21.

26. Cerda H. Los elementos de la investigación. Bogotá, Colombia: Editorial El Búho. 2002.
27. Hernández R, Fernández C, Batista P. Metodología de la investigación (5ae ed.) México: McGraw-Hill; 2003.

28. Korkman M. Introduction to the special issue on normal neuropsychological development in the school-age years. Developmental Neuropsychology. 2001;20:325330.

29. George D, Mallery P. SPSS For Windows step by step: A

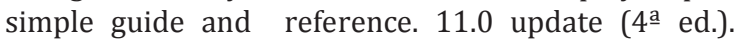
Boston, USA: Allyn \& Bacon. 2003.

30. Portellano J, Mateos R, Granados J, Tapia A, Ramos J, Pariente V, Martínez R. Prevención del fracaso escolar. Premios nacionales de investigación e innovación educativa. 1996;1:103-116.

31. Rosselli M, Matute E, Ardila A. Neuropsicología del desarrollo infantil. México DF, México: Editorial Manual Moderno. 2010.

32. Vilatuña M. Evaluación Neuropsicológica en niños de cuatro a cinco años con problemas de lenguaje. Quito. [Tesis de maestría]. 2012.

33. Aponte M, Zapata M. Caracterización de las funciones cognitivas de un grupo de estudiantes con trastornos específicos del aprendizaje en un colegio de la ciudad de Cali, Colombia. Psicología: Avances de la disciplina. 2013;7(1):23-34.

34. Escurra L, Matalinares M, Cuzcano A, Panca N, Manruique E, Varillas R, Polido S. Las habilidades metalingüísticas y el rendimiento lector en dos grupos de alumnos de condición socioeconómica baja que cursan el 1er grado de primaria en colegios públicos y privados de San Juan de Lurigancho. Revista de Investigación en Psicología. 2002;5 (1):27-34.

35. Portellano J. Neuropsicología infantil. Madrid, España: Editorial Síntesis. 2007.

36. Solovieva Y, Quintanar L. Antología del desarrollo psicológico del niño en edad preescolar. México DF, México: Editorial Trillas. 2010.

37. Pérez M, Escotto E, Arango J, Quintanar L. Rehabilitación neuropsicológica. Estrategias en trastornos de la infancia y el adulto. México DF, México: Manual Moderno. 2014.

38. Baeza R, Rodríguez D. Comprensión de la prosodia en el lenguaje verbal de niños escolares. Revista de Educación y Desarrollo. 2011;17:5-14.

39. Feldman R. Desarrollo en la infancia. México DF, México: Editorial Pearson. 2008.

40. García L, Pérez M. Viso percepción. Neuritas. Evaluación y rehabilitación neuropsicológica. 2010.

41. Portellano J, García J. Neuropsicología de la atención, las funciones ejecutivas y la memoria. Madrid, España: Editorial Síntesis. 2014.

42. Portellano J. Introducción a la neuropsicología. Madrid, España: Editorial McGraw Hill. 2005.

43. Fernandes M, Bentes $R$, Figueiredo $P$, da CostaAbrantes, Parizzi A, Borges W, Salgado J. Normalización de una batería de tests para evaluar las habilidades de comprensión del lenguaje, fluidez verbal y denominación en niños brasileños de 7 a 10 años: resultados preliminares. Revista de Neurología. 2007;44(5):275-280. 
44. Gasca C, Pallarés J. Aspectos psicolingüísticos en el síndrome del cromosoma $\mathrm{X}$ frágil. Revista de Neurología. 2001;33(1):29- 32.

45. Matute E, Rosselli M, Ardila A, Morales G. Verbal and nonverbal fluency in spanish - speaking children. Developmental Neuropsychology. 2004;26:647-660.

46. Phillips S, Steele S, Tanz C. Lengua, género y sexo desde una perspectiva comparada. México DF, México: Editorial Abya Yala. 1999.

47. Abad S, Brusasca M, Labiano L. Neuropsicología infantil y educación especial. Revista Intercontinental de Psicología y Educación. 2009;11(1):199-216.

48. Muñoz M, Tirapu J. Rehabilitación Neuropsicológica. Madrid, España: Editorial Síntesis. 2008.

49. Arruabarrena I, De Paúl J. Early intervention programs for children and families: theoretical and empirical bases supporting their social and economic efficiency. Psychosocial Intervention. 2012;21(2):117-127.

50. González MC, Solovieva Y, Quintanar L. El juego temático de roles sociales: aportes al desarrollo en la edad preescolar. El juego temático de roles sociales: aportes al desarrollo en la edad preescolar. Avances en Psicología Latinoamericana. 2014; 32(2):287-308.

51. Quintanar L, Solovieva Y. Métodos de corrección neuropsicológica en preescolares mexicanos con TDA. Revista de Psicología General. 2006;4(11):6-15. 